\title{
Investigação da alteração hidrotermal por meio de técnicas de PDI e SIG, no Distrito Fluorítico de Santa Catarina (DFSC), Brasil
}

\author{
Rosemary Hoff ${ }^{1}$ \& Artur Cezar Bastos Neto ${ }^{2}$
}

\begin{abstract}
Resumo O Distrito Fluorítico de Santa Catarina (DFSC) é o maior produtor de fluorita da América do Sul. Atualmente, tem sua produção estabilizada devido ao custo do minério produzido no país ser igual ou menor ao minério importado, havendo necessidade de ampliação das reservas que alcançam 2,2 milhões de toneladas. A fluorita ocorre em filões sub-verticais, média de 3 metros de largura por 400 metros de comprimento, controlados por falhas e fraturas que se estendem por quilômetros e a alteração hidrotermal ocorre numa faixa de até 100 metros de largura em torno dos filões. Neste estudo, o enfoque da prospecção de fluorita foi feito pela integração de dados estruturais e de alteração hidrotermal. O objetivo deste trabalho foi a aplicação de técnicas de processamento digital de imagens (PDI) e de sistema de informação geográfica (SIG) sobre dados TM LANDSAT 5 para distinguir a alteração hidrotermal. As morfo-estruturas foram obtidas pela filtragem multidirecional da banda TM4, as quais foram digitalizadas e depois classificadas conforme os intervalos azimutais correspondentes às direções de interesse para a prospecção dos filões de fluorita. Para realçar feições de alteração hidrotermal, foram aplicadas razões entre bandas como 3/1, 5/1 e 5/4 para discriminar concentrações de óxidos e hidróxidos de ferro; 5/7 para argilo-minerais e 1/7 para a alteração hidrotermal em geral. As imagens em níveis de cinza não realçaram muito bem o resultado, por isto foi feita reclassificação baseada no comportamento espectral do material. Estes dados foram cruzados com dados de estruturais por meio de técnicas de SIG, sendo feitos mapa de distâncias de 100 metros a partir das morfo-estruturas, os quais foram multiplicados pelas imagens de alteração hidrotermal. Finalmente, foram delimitadas zonas mais favoráveis à prospecção de fluorita dentro do distrito, baseadas na maior densidade de pixels de alteração hidrotermal restritos ao entorno de 100 metros das morfo-estruturas.
\end{abstract}

Palavras-chave: alteração hidrotermal, sensoriamento remoto geológico, SIG, fluorita.

\begin{abstract}
Reaserch on hydrothermal alteration, by DIP and GIS techniques, in Santa Catarina Fluorine District, Brazil. Santa Catarina Fluorite District (DFSC) is the major producer of South America, and currently production has stabilized due the ore produced cost in Brazil is equal or smaller than the imported ore price. However, DFSC needs to increase reservations. The fluorite occurs in sub-vertical veins, 3 meters width by 400 meters length. The beds are controlled by faults and fractures that extend themselves for kilometers, and the hydrothermal alteration occurs in strips up to 100 meters width from the veins. The fluorite search in DFSC should be make by integration of structural data and alteration. The work objective was developing techniques to distinguish the hydrothermal alteration by application digital image processing (DIP) and Geographic Information System (GIS) techniques on TM LANDSAT 5 data. The morpho-structures were obtained by filtering TM4 band, after digitalized and classified according to azimuth intervals related to the fluorite veins. To enhance alteration feature, $3 / 1,5 / 1$ and $5 / 4$ band ratio was employed to discriminate iron oxide and hydroxide concentrations; $5 / 7$ for clay mineral and 1/7 for general alteration. Grayscale images not enhanced the result and than reclassification was made based on their spectral behavior. SIG techniques crossed these data with structural vectors. It generated distance map around morphostructures and were multiplied by hydrothermal alteration images. Finally, more favorable zones were delimited, based on the pixel density of alteration around 100 meters of morphostructures.
\end{abstract}

Keywords: hydrothermal alteration, geological remote sensing, GIS, fluorite.

INTRODUÇÃO O Distrito Fluorítico de Santa Catarina (DFSC) é o maior produtor de fluorita da América do Sul. Cerca de $3 \mathrm{Mt}$, ROM (run of mine) com teor médio de $40 \%$ de $\mathrm{CaF} 2$ ) foram produzidas em 40 anos de exploração contínua, cujo auge (300.000 t/ano) aconteceu na década de 80 . Na última década, a pesquisa mineral por novos depósitos estagnou, mas a produ- ção nacional permaneceu concentrada no DFSC, onde as reservas atuais são cerca de $2 \mathrm{Mt}$ (minério In situ com $50 \%$ de CaF2). Assim, diante de uma necessidade premente, a pesquisa vem sendo retomada.

Neste estudo o objetivo foi a integração de dados texturais e espectrais das imagens de satélite TM LANDSAT 5, utilizando-se produtos gerados pela filtragem

1 - Empresa Brasileira de Pesquisa Agropecuária, Bento Gonçalves (RS), Brasil. E-mail: rosehoff@cnpuv.embrapa.br

2 - Instituto de Geociências, Universidade Federal do Rio Grande do Sul, Porto Alegre (RS), Brasil. E-mail: artur.bastos@ufrgs.br 
e seus vetores derivados, que são as morfo-estruturas (Hoff et al. 2002), bem como produtos raster gerados por razões entre bandas e reclassificação de imagem, que é a alteração hidrotermal. A integração dos dados foi feita pela aplicação de técnicas de SIG.

Os objetivos específicos do trabalho foram a identificação e classificação espacial das morfo-estruturas favoráveis à deposição da fluorita; a caracterização da alteração hidrotermal e sua distribuição no distrito e o desenvolvimento de técnicas de processamento digital de imagens e SIG, capazes de integrar morfo-estruturas com alteração hidrotermal. O objetivo geral é a ampliação do conhecimento geológico do distrito visando a prospecção de fluorita.

TRABALHOSANTERIORES ODFSC ocorre numa faixa de $100 \mathrm{~km}$ de comprimento por $60 \mathrm{~km}$ de largura, delimitada a oeste pela bacia do Paraná e a leste pelo Oceano Atlântico (Fig. 1). O embasamento enquadra-se na extremidade nordeste do Batólito de Pelotas - unidade geotectônica central do Cinturão Dom Feliciano (Fragoso César 1980). Predominam os granitos Pedras Grandes e Tabuleiro (Sallet 1988, Zanini et al. 1997, Silva et al.
1999) identificaram no distrito faixas com ocorrências da Suíte Valsungana. Os granitóides são cortados por diques de rochas sub-vulcânicas ácidas eo-cambrianas (Teixeira 1969). As rochas da Bacia do Paraná presentes no distrito pertencem às formações Rio do Sul (folhelhos e diamictitos) e Rio Bonito (arenitos, siltitos e carvão), ambas do Permiano Inferior. As rochas acima são cortadas por diques e soleiras de diabásio da Formação Serra Geral (juro-cretácea). Na parte norte do distrito ocorre a intrusão alcalina de Anitápolis e, a oeste, o maciço alcalino de Lages com $70 \mathrm{Ma}$ (Scheibe 1986).

A principal estrutura com mineralizações associadas é o lineamento Canela Grande (LCG) de direção NNE-SSW com mais de $100 \mathrm{~km}$ de comprimento e até $2 \mathrm{~km}$ de largura. O LCG é constituído por um sistema de falhas de direções N-S e NNE-SSW (subordinadamente NE-SW), ocorrendo estruturas associadas menores de direção NE-SW a E-W e vinculadas à mineralização. Segundo Hackspaker \& Flores 1987, Ferreira \& Almeida 1988, Bastos Neto 1990, a fluorita pode ter sido controlada por falhas principais ou pelas estruturas menores associadas.

Ao longo do LCG situam-se 4 sub-distritos mi-

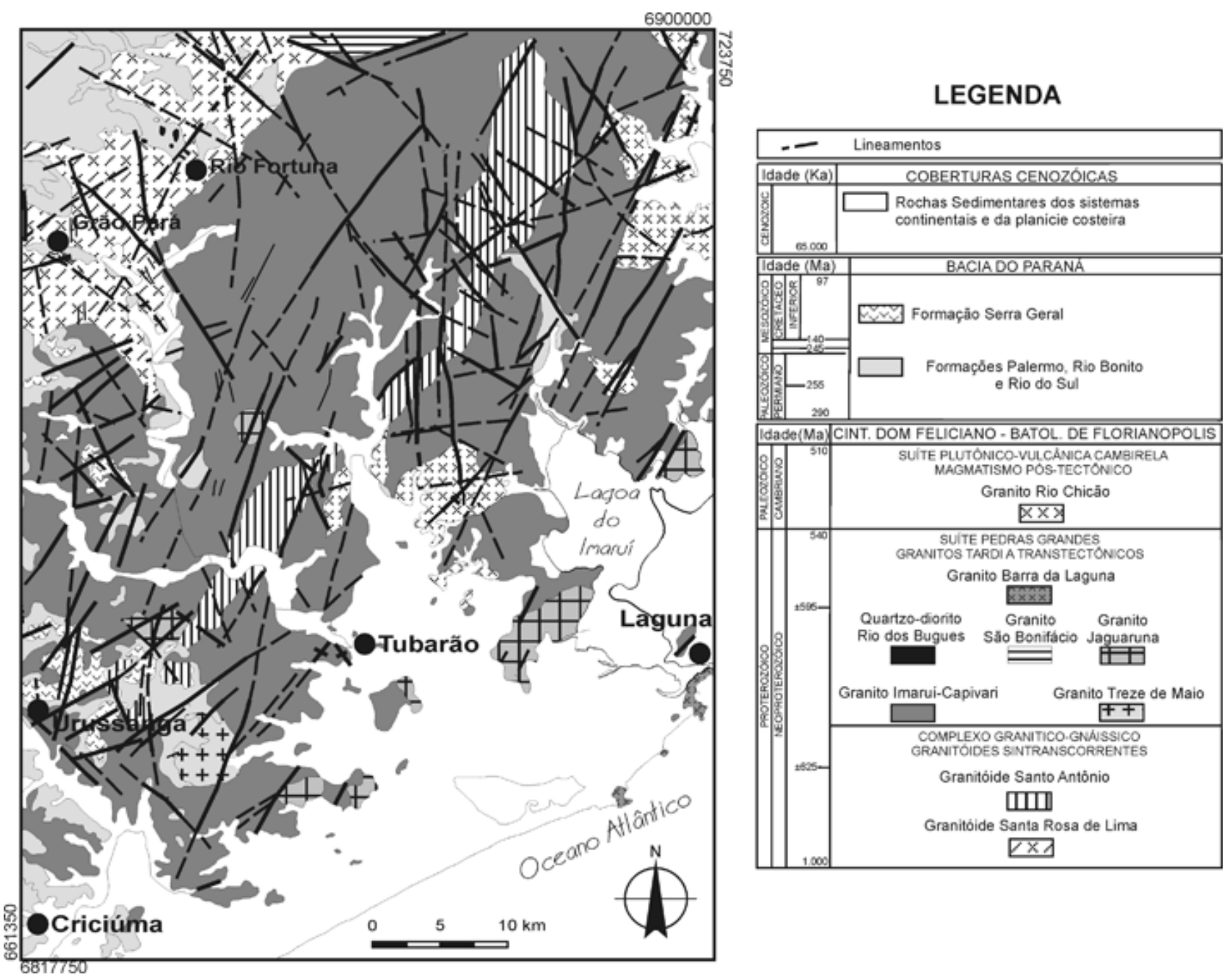

Figura 1 - Mapa geológico do DFSC, modificado de Silva \& Leites 2000. 
neiros, sendo que as reservas atuais concentram-se no sub-distrito Grão-Pará, associados à zona de cisalhamento Rio dos Bugres (ZCRB) (Ferreira \& Almeida, 1988). A leste do LCG foram explorados depósitos associados ao lineamento Armazém e os depósitos Garganta e São Tomás. Os depósitos são filoneanos hidrotermais de baixa temperatura, a paragênese é formada essencialmente por fluorita e quartzo microcristalino, subordinadamente barita, pirita e esmectita e, mais raramente, galena e carbonatos. Segundo Bastos Neto 1990, a mineralização no LCG foi ligada a quatro fases tectônicas, sendo que os modelos genéticos propõem a vinculação da mineralização ao magmatismo alcalino de Lages (Horbach \& Marimon 1982) ou ao magmatismo alcalino de Anitápolis (Morgental 1984). Este autor considera que o flúor foi lixiviado dos granitos regionais por soluções de origem meteórica (Savi 1980, Dardenne \& Savi 1984, Dardenne 1985, Sallet 1988, Ferreira \& Almeida 1988, Bastos Neto 1990, Bastos Neto et al. 1991, Rocha 1997).

Técnicas de sensoriamento remoto foram algumas vezes empregadas no distrito, inicialmente Horbach \& Marimon 1982 identificaram as principais estruturas de distrito a partir de imagens de radar. Morgental \& Kirchner 1983 obtiveram as estruturas do distrito por fotografias aéreas e imagens de radar. Ferreira \& Almeida 1988 utilizaram fotografias aéreas e imagens TM LANDSAT 4 em estudos na ZCRB e extremo no sul do LCG. Flores et al. 1993 utilizaram imagens TM LANDSAT 5 e fotografias aéreas, definindo áreas com diferentes padrões morfo-tectônicos e morfogenéticos para diferentes potenciais para prospecção de fluorita. Hoff 2002 e Hoff et al. 2002 estudaram detalhadamente as morfo-estruturas do DFSC a partir de dados aeromagnetométricos comparando-as com a ocorrência da alteração hidrotermal dentro dos corpos graníticos e Hoff et al. 2004 estudaram a ocorrência de alteração hidrotermal por meio de dados aerogamaespectrométricos comparados com dados orbitais TM LANDSAT 5.

MATERIAS E MÉTODOS A imagem utilizada no presente estudo foi TM LANDSAT 5, WRS 220.079 de 23.05.97 (GLCF, .2008), e os processamentos de imagem foram feitos no programa ENVI 4.1 (RSI 2008); digitalização no Autocad MAP (Autodesk, 2008) e a análise vetorial no Rockworks 14 (Rockware, 2008). A base geológica utilizou o mapa geológico da Folha Criciúma executado pelo Serviço Geológico do Brasil - CPRM (Silva \& Leites 2000). A base de dados e os dados orbitais foram georreferenciados pela coleta de controle de pontos no terreno com apoio de GPS (global position system). Este trabalho apresenta os resultados do desenvolvimento técnicas para integrar dados de morfo-estruturas (vetoriais) obtidos pelos atributos texturais e dados de alteração hidrotermal fornecidos pelas características espectrais de imagens orbitais, por meio de técnicas de SIG detalhadas em Hoff 2002.

$\mathrm{O}$ aumento linear de contraste foi efetuado para obtenção do histograma expandido de cada banda, com a finalidade de eliminar problemas gerados pelas con- dições de iluminação da cena que correspondem ao sombreamento e à contribuição atmosférica (Richards 1986). Para melhorar o aspecto visual, foi aplicado o aumento linear de contraste - stretch.

Filtragens A técnica utilizada para detecção de lineamentos estruturais foi a filtragem direcional sobre a banda TM4, escolhida pela sua definição dos elementos do relevo, atenuação dos efeitos da vegetação (alta reflectividade) e, conseqüentemente, melhor visualização dos lineamentos. A filtragem direcional foi feita por aplicação de filtro multidirecional 7 x 7 (Araújo \& Carvalho Jr. 1994). Os lineamentos foram digitalizados sobre a imagem filtrada, produzindo-se arquivo de dados contendo mais de 16.000 vetores. A análise espacial dos dados vetoriais calculou a direção azimutal dos vetores e os classificou segundo intervalos de interesse para a fluorita.

Razões entre bandas Estas operações aritméticas foram utilizadas para reduzir os efeitos que degradam a informação espectral, genericamente denominados efeitos ambientais como por exemplo, os efeitos topográficos e realçar a informação espectral de interesse, como a concentração anômala de óxidos e hidróxidos de ferro e argilo-minerais. As razões 3/1, 5/1 e 5/4 foram escolhidas em função das concentrações anômalas de óxidos e hidróxidos de ferro, que normalmente apresentam absorções nas bandas 1 e 4 e maior reflectância nas bandas 3 e 5. A razão 5/7 é indicada para revelar a presença de argilo-minerais, caracterizada pela reflectância na banda 5 e absorção na banda 7 . A razão $1 / 7$ buscou discriminar os produtos de alteração hidrotermal de forma conjunta, de modo que as concentrações destes resultem em valores próximos de 1, enquanto que as concentrações de óxidos em relação à concentração de argilo-minerais tenham valores menores que 1. A razão $4 / 3$ foi utilizada para minimizar os efeitos da cobertura vegetal sobre a informação espectral dos solos e rochas conforme utilizado por Ferreira Jr. 1993 e Klein 1998. Baseando-se nestas premissas, foram testadas razões compostas do tipo $\mathrm{A}-\mathrm{B} / \mathrm{A}+\mathrm{B}$, para obtenção de produtos com variância mais baixa que as razões simples, visando a detecção de feições sutis de concentrações de óxidos de ferro e argilo-minerais.

Reclassificação A partir do estudo do comportamento espectral destes materiais em cada banda TM LANDSAT 5 e baseando-se no histograma da imagens, desenvolveu-se um método focado para a investigação de zonas de alteração hidrotermal, sendo a reclassificação de produtos de razão entre as bandas originais. Isto consiste na atribuição de valores de zero para os NDs (números digitais) que não interessam e valor um para os valores de NDs que representam os alvos prospectados, como óxidos de ferro e argilo-minerais.

Técnicas de SIG A partir dos dados vetoriais de morfo-estruturas separados por intervalos de classe, foram feitos mapas de distâncias das morfo-estruturas, reclassificados para distância de 100 metros no entorno das 
mesmas. Esta medida foi escolhida baseado no caso do Filão Rio Bravo Alto (Bastos Neto, 1990) no norte do DFSC que apresenta alteração hidrotermal nos granitos encaixantes da mineralização numa faixa que se estende por aproximadamente $100 \mathrm{~m}$ no entorno do filão de fluorita. Os mapas de distância de 100 m são imagens boleanas que foram multiplicadas pela imagem de alteração hidrotermal, criando-se novas imagens em que a alteração hidrotermal se restringe ao entorno de 100 metros das morfo-estruturas (Fig. 2).

\section{RESULTADOS}

Filtragens A banda TM4 mostra formas de relevo bem definidas e os corpos d'água em contraste, tendo ND próximo ou igual a zero, muito discrepante das outras bandas. A maior reflectância da vegetação atenuou o contraste do uso do solo, evidenciando melhor os elementos do relevo (Fig. 3 a). Consequentemente, a visualização dos lineamentos melhora bastante, revelando as morfo-estruturas. (Fig. 3 b).

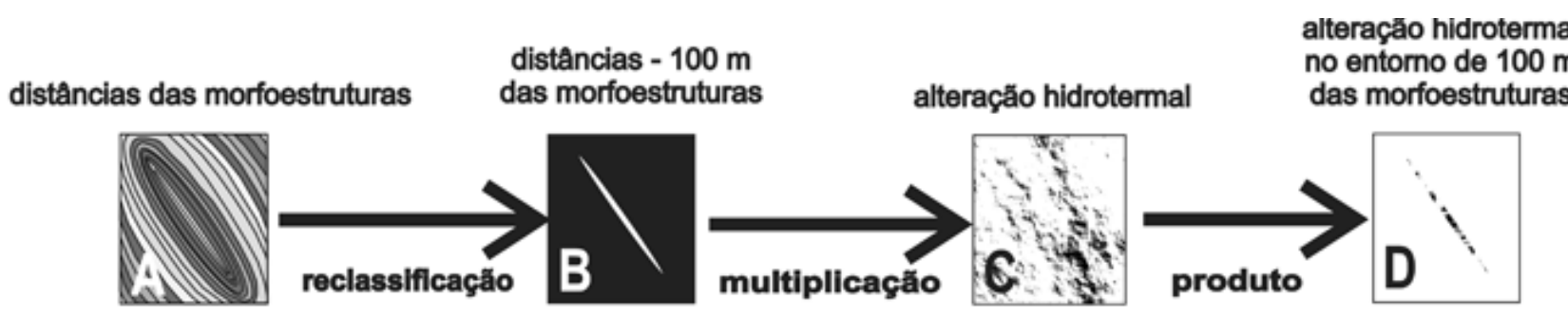

Figura 2 - Esquema de integração de dados de morfo-estruturas e de alteração hidrotermal por meio de técnicas de SIG.
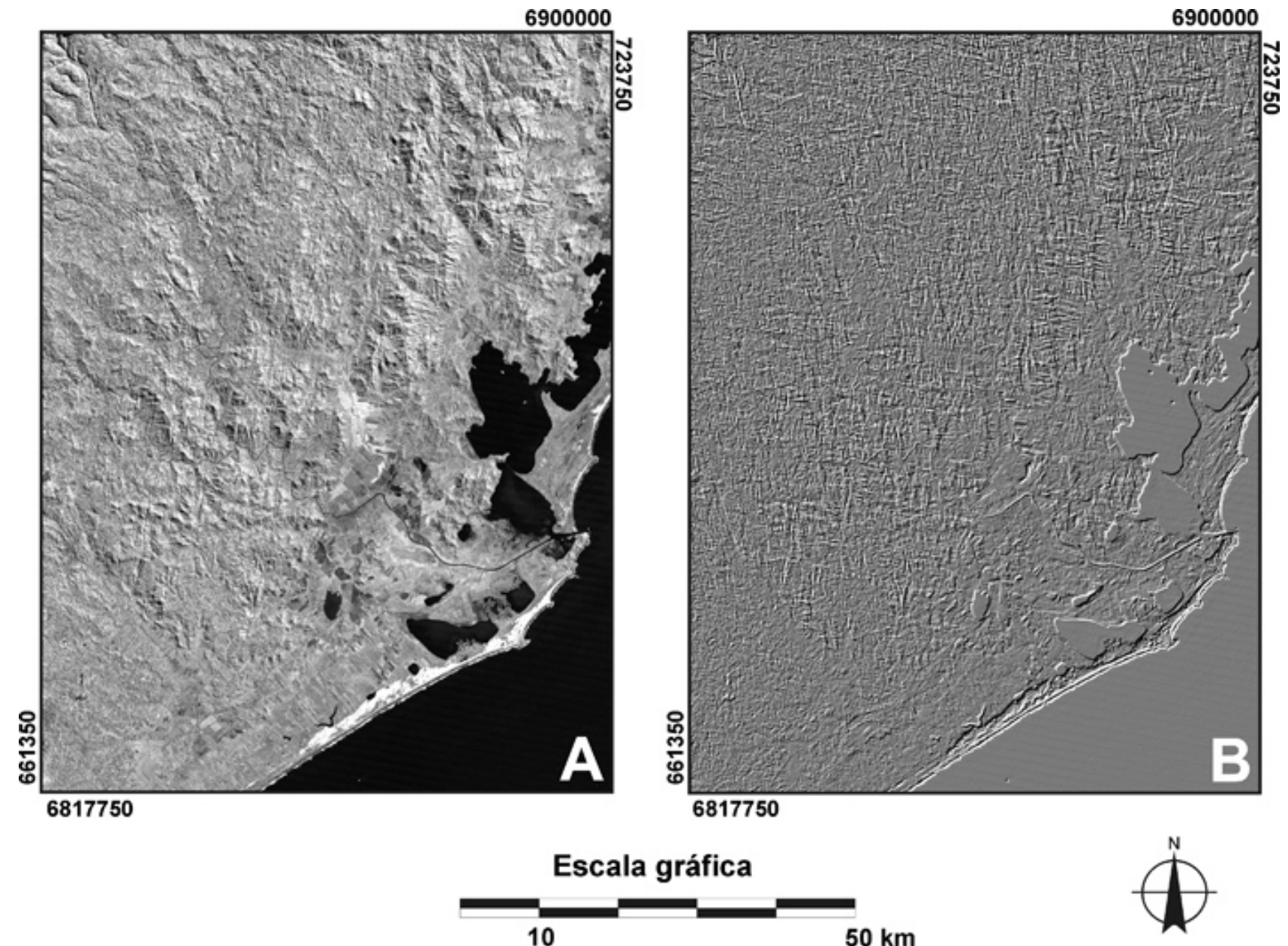

Figura 3 - Imagens TM LANDSAT 5: Banda TM4 com aumento linear de contraste (a) e Banda TM4 com aplicação de filtro multi-direcional (b). 
Análise espacial dos vetores Os lineamentos do DFSC foram digitalizados sobre a imagem filtrada, excluindo-se a região da planície costeira e grandes corpos aluvionares (zonas onde há pouca possibilidade de haver morfo-estruturas mineralizadas), compondo um arquivo de dados vetoriais, obtendo-se uma população em torno de 16.000 feições lineares (Fig. 4 a).

A distribuição das morfo-estruturas foi investigada segundo intervalos de direções de interesse para fluorita. No quadrante NE, as subdivisões $0^{\circ}-20^{\circ}, 20^{\circ}$ $45^{\circ}$ e $45^{\circ}-80^{\circ}$ correspondem às relações angulares das estruturas que constituem o LCG (Fig. 4). Este último intervalo foi delimitado em função da direção do filão Cruzeiro $\left(\mathrm{N} 80^{\circ} \mathrm{E}\right)$ e representa o limite angular para os depósitos de fluorita conhecidos. O intervalo $45^{\circ}-80^{\circ}$ permite investigar também os lineamentos da família ENE-WSW e as estruturas menores a estes associadas, cuja importância na compartimentação tectônica da área é reconhecida por Horbach e Marimon 1982 e Morgental 1984. As relações com a distribuição da mineralização de fluorita já foram propostas por Bastos Neto 1990 e Flores 1998. Os intervalos N80 $-90^{\circ} \mathrm{E}$ e $\mathrm{N} 80^{\circ}-90^{\circ} \mathrm{W}$ permitem avaliar a presença no continente de estruturas ligadas ao lineamento de Florianópolis ou estrutura oceânica E-W (Asmus 1984) a qual seria a responsável pela reativação das estruturas NNE-SSW controladoras dos depósitos de fluorita do DFSC (Horbach e Marimon 1982 e Morgental 1984).

Razões entre bandas Entre as razões de bandas testadas por Hoff 2002, nas quais foi investigada a alteração hidrotermal, considerou-se que para a razão $1 / 7$ os pixels claros corresponderam a argilo-minerais, enquanto que as áreas escuras são atribuídas aos óxidos de ferro, podendo ser confundidos com de corpos d'água e dunas, respectivamente. Porém, nesta razão observou-se a separação por tons de cinza de dois importantes grupos de alteração hidrotermal, os óxidos de ferro e argilominerais (Fig. 5a). As concentrações destes minerais em determinadas áreas podem ser comparadas com resultados de Hoff et al. 2004 sobre dados aerogamaespectrométricos no DFSC.

Reclassificação A imagem da razão entre as bandas 1/7 reclassificada (Fig. 5a) exibiu um resultado bastante interessante para investigação de argilo-minerais,

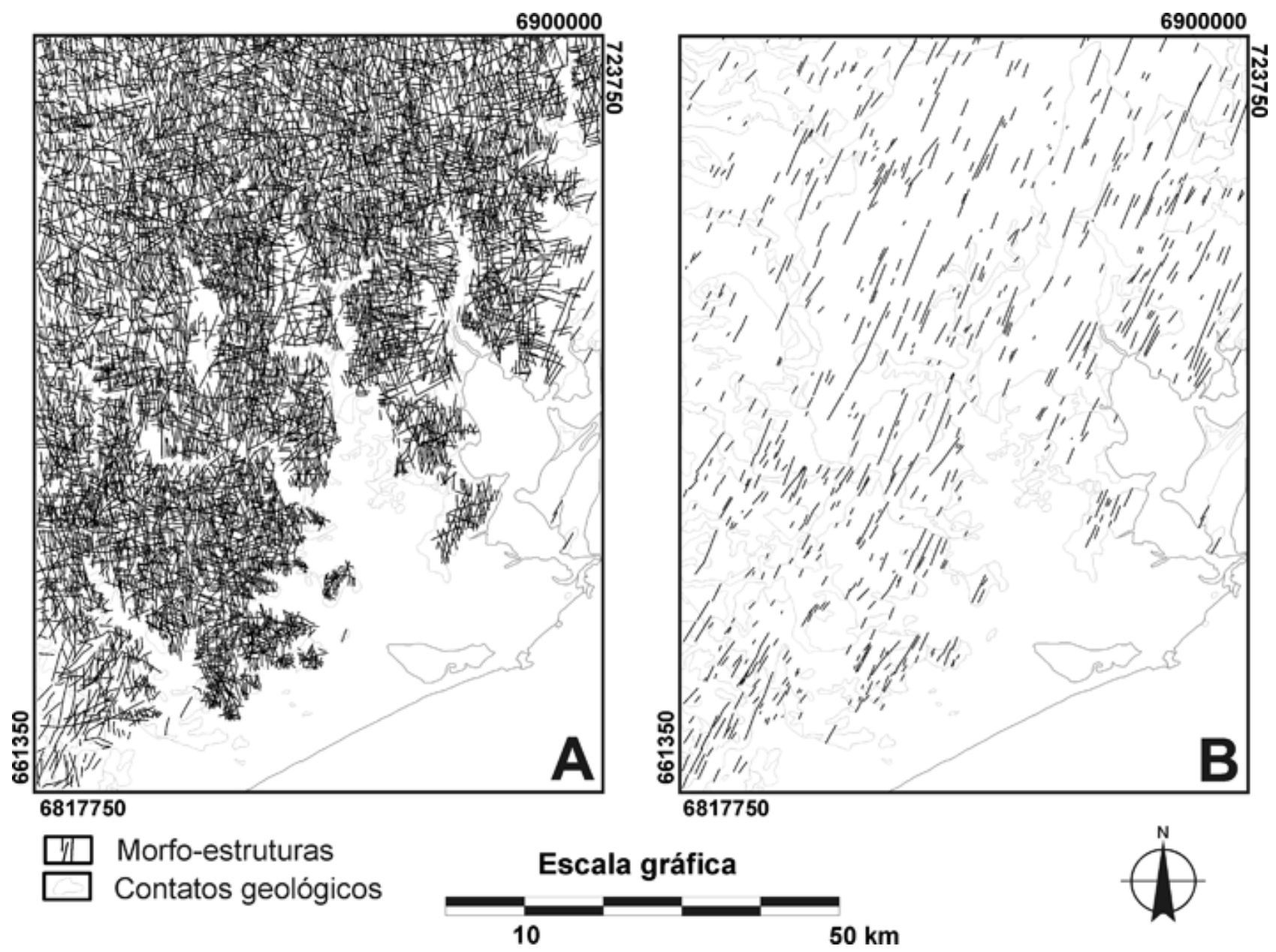

Figura 4 - Morfo-estruturas do DFSC com a população total (A) e sub-divisão de morfo-estruturas de interesse para a fluorita no DFSC, $N 20^{\circ}-45^{\circ} \mathrm{E}(\mathrm{B})$. 


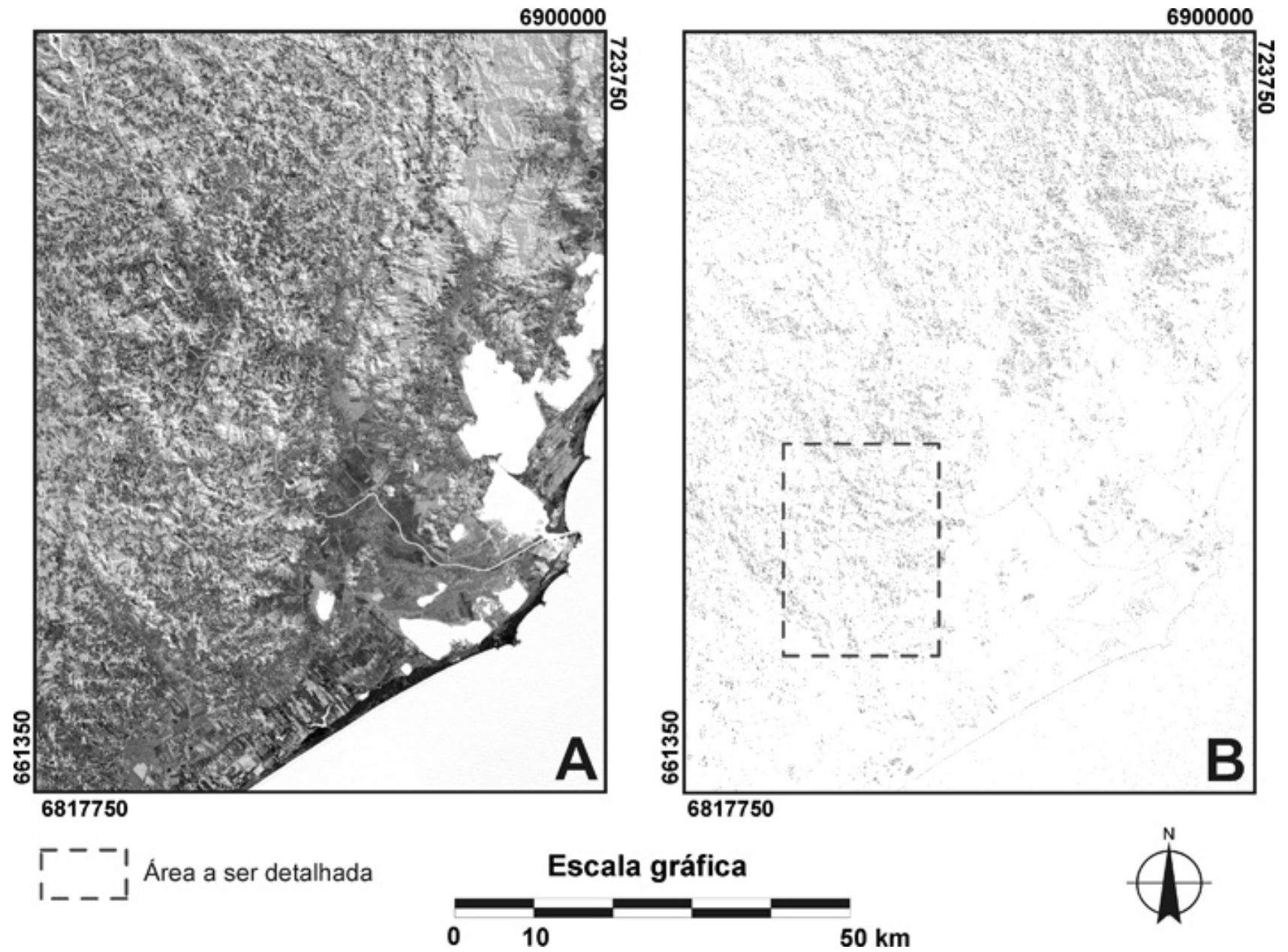

Figura 5 - Razão entre bandas TM1/TM7 (A) e imagem reclassificada para argilo-minerais (B), mostrando área a ser destacada pelo interesse em ocorrências conhecidas de fluorita.

realçando-se feições dentro um contexto geral de distribuição dos pixels em faixas de direção NW-SE e pela existência de alinhamentos de menor expressão em direções do quadrante NE. Além disto, o zoneamento geral dos pixels correspondente a alteração hidrotermal assemelha-se aos dados aerogamaespectrométricos processados por Hoff et al. 2004.

No detalhe da área situado próximo a Urussanga (Fig. 6) foram assinalados alguns destes alinhamentos de pixels como: grupo de pixels ocorrem alinhados e no conjunto encontram-se isolados; múltiplos pixels alinhados e totalmente independentes das faixas NWSE; alinhamentos de múltiplos pixels internos a uma faixa NW-SE e; alinhamentos que atravessam as faixas NW-SE. Percebe-se entre os exemplos assinalados, que diferentes alinhamentos podem estar espacialmente relacionados, formando um alinhamento de maior extensão, como é o caso na parte central da figura. A esta figura foram superpostas as morfo-estruturas N-S a NNE-SSW, percebendo-se que alguns dos alinhamentos antes assinalados não apresentam correspondência com as morfo-estruturas e, do mesmo modo, inúmeras morfo-estruturas não têm pixels associados. Por outro lado, há casos de superposições excelentes, como o as- sinalado, onde uma morfo-estrurura relativamente extensa apresenta na sua parte intermediária uma ligeira inflexão para $\mathrm{E}$ (zona propícia para a abertura de caixa filoneana) e exatamente nesta inflexão ocorre uma grande concentração de pixels. Por outro lado, confirma-se que existem diversos alinhamentos de pixels com morfo-estruturas associadas nas faixas NW-SE.

Integração das morfo-estruturas com imagem de alteração hidrotermal A integração dos dados de morfo-estruturas com a imagem de razão 1/7 reclassificada baseou-se nos testes de superposição de famílias de morfo-estruturas de interesse para fluorita sobre a imagem de alteração hidrotermal. Para analisar o comportamento da alteração hidrotermal no entorno das morfo-estruturas, delimitou-se como área de interesse uma faixa de $100 \mathrm{~m}$ de cada lado da morfo-estrutura. Este tipo de cruzamento de dados realça os alinhamentos de pixels segundo as direções de cada família de morfo-estruturas, tendo como critério o alinhamento e a densidade dos pixels, segundo as etapas de execução: (a) mapas de distâncias das morfo-estruturas, a partir da rasterização dos vetores; (b) mapas de distância de $100 \mathrm{~m}$, a partir da reclassificação do mapa de distância; (c) mapa de alteração hidrotermal 

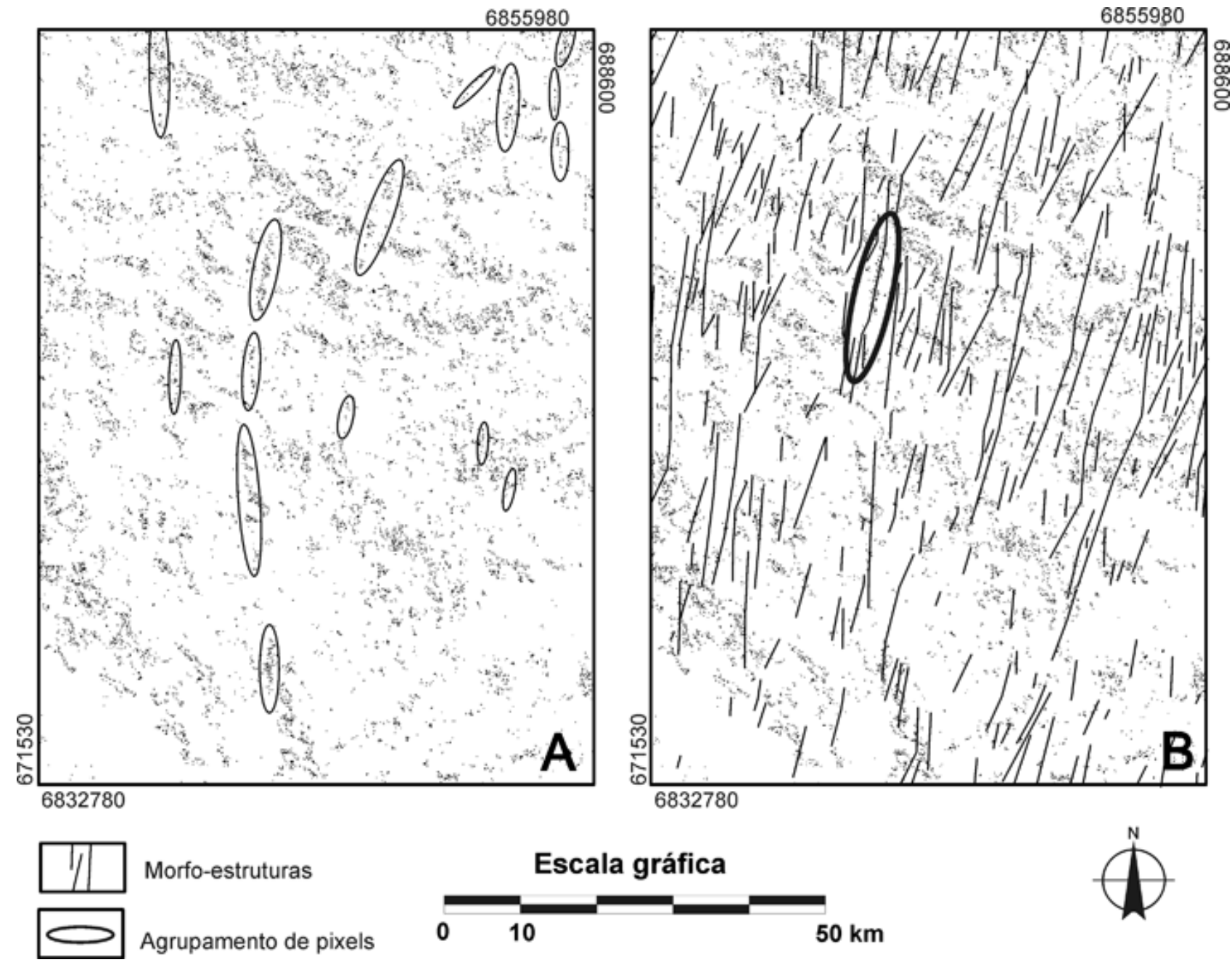

Morfo-estruturas
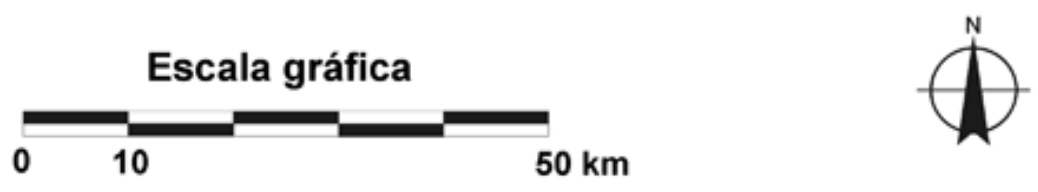

Agrupamento de pixels

Figura 6 - Detalhe da Razão entre bandas TM1/TM7 - TM LANDSAT 5 reclassificada para obter feições relativas a argilo-minerais, mostrando os agrupamentos dos pixels (A) e com as morfo-estruturas sobrepostas (B).

no entorno de $100 \mathrm{~m}$ das morfo-estruturas, por meio da multiplicação do mapa de distância de 100 m e a imagem de alteração hidrotermal.

As imagens de alteração comparadas com as estruturas correspondentes, percebe-se que o número de morfo-estruturas é incomparavelmente maior do que os alinhamentos de pixels relacionados à alteração hidrotermal. Apesar da boa distribuição de pixels na imagem, nem todas as morfo-estruturas mapeadas têm pixels associados.

O cruzamento de dados representa uma seleção automática prévia das morfo-estruturas prospectáveis e as imagens produzidas pela integração mostraram alinhamentos de pixels com extensão e densidade variáveis. Nos casos de alinhamentos maiores, possivelmente trata-se de alteração hidrotermal associadas às morfo-estruturas e concentrações localizadas de pixels podem estar relacionadas a cruzamentos com estruturas de outras direções.

Este é um aspecto da geologia da fluorita do DFSC que pode ser observado na escala de superfície e nas minas de fluorita, ou seja, o aumento do efeito da alteração hidrotermal nos cruzamentos de estruturas. Estes pontos de maiores concentrações tendem a distribuir-se em faixas de ocorrência das grandes morfoestruturas WNW-ESE.

Em outros locais, observaram-se concentrações de pixels numa espessura que ultrapassa a largura estabelecida às margens de cada morfo-estrutura. Esta configuração deve-se à existência de morfo-estruturas paralelas muito próximas a bifurcações ou interseções de estruturas com pequenas diferenças angulares, sendo sítios altamente prospectáveis em vista a formação de zonas favoráveis à abertura de caixas filoneanas (Morgental 1984, Ferreira \& Almeida 1988).

No caso do cruzamento de dados para o intervalo $\mathrm{N} 20^{\circ}-45^{\circ} \mathrm{E}$, o número de alinhamentos de pixels é muito semelhante ao de morfo-estruturas. Por outro lado, é bastante grande o número de alinhamentos com grande densidade de pixels ocupando em muitos casos toda a largura da faixa estabelecida, porque este é o intervalo direcional da grande maioria dos filões de fluorita do DFSC. No que se refere aos sítios prospectáveis, observou-se a existência de áreas relativamente 
bem definidas onde os alinhamentos de pixels são mais freqüentes, individualmente mais densos e mais largos. Assim, analisando o agrupamento dos pixels na figura 7, pode-se observar o seguinte:

(1) Área A - uma concentração a nordeste do DFSC, porém pouco prospectável, pois esta quantidade excessiva de pixels pode estar relacionada a óxidos de ferro dos diques de diabásio, observados por Hoff et al. 2002 a partir de dados magnetométricos;

(2) Área B - uma faixa ao longo do eixo central do DFSC (NNE-SSW), destacando-se no extremo norte desta faixa alinhamentos com inflexões e zonas de bifurcação, coincidindo com dados de alteração hidrotermal obtidos por $\mathrm{F}$ apontados por Hoff 2002;

(3) Ârea C - uma área de direção WNW-ESSE contendo alinhamentos NNE-SSW espessos relacionados a interseções com estruturas WNW-ESE;

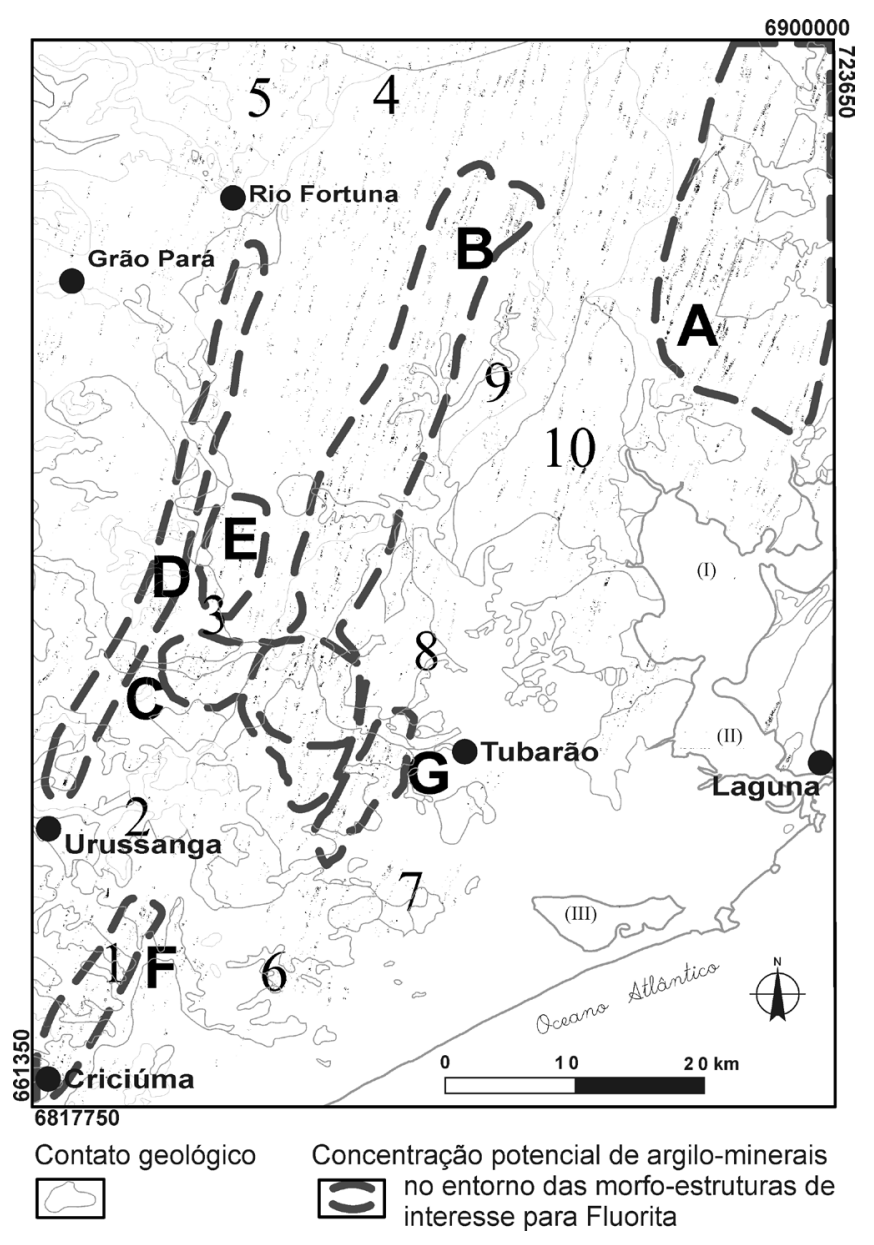

Figura 7 - Multiplicação da imagem de distância das morfo-estruturas $N 20^{\circ}-45^{\circ} \mathrm{E}$ com a imagem reclassificada para argilo-minerais, destacando de $A$ a $G$ áreas de interesse para fluorita. Ocorrências de fluorita nos sub-distritos: 1 - Segunda Linha Torrens, 2 - Ribeirão da Areia, 3 - Pedras Grandes, 4 - Rio Bravo Alto, 5 Grão Pará e nos filões: 6-Garganta, 7 -Jaguaruna, 8 - São Martinho, 9 -Armazém, 10 - São Tomás.

(I) - Lagoa do Imarui, (II) - Lagoa Santo Antônio, (III) - Lagoa Garopaba do Sul.
(4) Área D - uma série de alinhamentos na direção NNE-SSW que correspondem ao lineamento identificado por Hoff et al. 2002, paralelo ao lineamento Canela Grande;

(5) Área E - alinhamentos coincidentes com o sub-distrito Pedras Grandes;

(6) Área F - uma concentração de pixels no sub-distrito Segunda Linha Torrens e uma série de alinhamentos relativamente densos no seu prolongamento para SSW deste sub-distrito;

(7) Área G - uma concentração de pixels no prolongamento para SW do Filão São Martinho.

A distribuição dos depósitos (e sub-distritos) ao longo dos lineamentos obedece, numa primeira escala, à localização em relação à rocha fonte. Mas, numa segunda escala, pode ter sido influenciada, também por uma compartimentação imposta pelas grandes estruturas ENE-WSW e WNW-ESE. As primeiras, como suposto por Bastos Neto 1990 podem ter relações com as zonas de inflexão das estruturas que compõem os lineamentos $\mathrm{N}-\mathrm{S}$ a NNE-SSW, assim como podem ter, em épocas posteriores à mineralização, controlado movimentações verticais de blocos responsáveis pela erosão de filões. As estruturas WNW-ESE podem ter exercido um papel fundamental no deslocamento dos fluidos hidrotermais e devem ser cuidadosamente consideradas na prospecção ao longo dos lineamentos mineralizados.

CONCLUSÕES E RECOMENDAÇÕES A aplicação de filtragem multidirecional para investigação de morfo-estruturas se mostrou bastante útil e, de uma forma geral, confirmou a presença das estruturas conhecidas, pertencentes aos principais lineamentos do DFSC. Além disto, revelou novas morfo-estruturas e se mostrou eficiente nas áreas onde a cobertura sedimentar da Bacia do Paraná mascara a ocorrência de estruturas mineralizadas, como o aparecimento do prolongamento do lineamento Canela Grande no Sub-distrito Segunda Linha Torrens, fato confirmado em campo.

De uma forma geral, a reclassificação de imagens aplicada nos produtos temáticos gerados por técnicas de razões entre bandas se mostrou eficiente no que diz respeito à análise qualitativa para investigação da alteração hidrotermal no DFSC.

A comparação com dados magnetométricos processados por Hoff et al. 2002 reforçou anomalias correspondentes às estruturas conhecidas no DFSC, como é o caso do lineamento Canela Grande e lineamento Armazém. E mostraram coerência com a distribuição alteração hidrotermal regional nos granitóides vistos por Hoff et al. 2004, em relação a dados aerogamaespectrométricos, permitindo a definição de áreas mais favoráveis para a prospecção de fluorita no distrito.

Recomenda-se a utilização de dados orbitais hiperespectrais na área do DFSC, utilizando imagens obtidas por sensores com maior resolução espectral do que o TM LANDSAT 5, visando a definição de melhores resultados no que diz respeito à prospecção de alteração hidrotermal.

$\mathrm{O}$ reconhecimento do prolongamento da ZCRB 
até a cidade de Urussanga, estrutura também detectada por aeromagnetometria e por produtos do processamento de imagem caracterizaram uma associação de morfo-estruturas ao longo de uma faixa com, pelo menos, $60 \mathrm{~km}$ de extensão para a qual foi proposta a designação lineamento Grão-Pará (Hoff 2002). Devido à sua localização adjacente à borda da Bacia do Paraná, este lineamento ao qual associam-se importantes depósitos de fluorita no sub-distrito Grão-Pará, foi recentemente exposto à erosão, considerando-se o principal prospecto do DFSC.

Agradecimentos À Universidade Federal do Rio Grande do Sul, ao CNPq, à Floral Mineração Ltda.

\section{Referências}

Araújo A.H. \& Carvalho Jr. O.A. 1994. Arquitetura e implementação computacional de filtros digitais multidirecionais para aplicação na detecção de lineamentos estruturais e contatos litológicos. In: Congresso Brasileiro de Geologia, 38, Camboriú (SC), Boletim de Resumos Expandidos, p. 355.

Asmus H.E. 1984. Geologia da margem continental brasileira. In: Schobbenhaus C., Campos D.A., Derze G.R. Asmus H.E. (eds.) Geologia do Brasil. Departamento Nacional da Produção Mineral, Brasília, Brasil, p. 443472.

AUTODESK (ed.) Autocad map 2008. Autodesk, Incorporation. Disponível em: http://www.autodesk.com. Acessado em 02.01.2002.

Bastos Neto A.C. 1990. Le district a fluorine de Santa Catarina (Brésil): mineralizations e alterations hydrothermales dans lens cadre geodynamique. These de doctorat en metallogenie, Université de Orléans, Orleans, 420p.

Bastos Neto A.C., Touray J.C., Charvet J.P., Dardenne M.A. (1991): Tectonic setting of vein deposits in the Santa Catarina fluorite district (S. Brazil). In: Pagel M. \& Leroy J.L. (eds.) Source Transport and Deposition of Metals. Balkema Rotterdam, p. 423-425.

Dardenne M.A. 1985. Os depósitos de fluorita da região sul do Brasil. In: Simpósio Sul-brasileiro de Geologia, 2, Florianópolis, SBG, Boletim de Resumos, p. 201-214.

Dardenne M.A. \& Savi C.N. 1984. Geologia e geoquímica dos filões de fluorita Segunda Linha Torrens e Cocal. Revista Brasileira de Geociências, 14(2):120-127.

Ferreira A.C. \& Almeida T.I.R. 1988. Tectônica transcorrente e imagens TM-LANDSAT aplicadas à prospecção de fluorita e barita em Santa Catarina. Revista Brasileira de Geociências, 19(2):207-223.

Ferreira Jr. L.G. 1993. Discriminação de produtos de alteração hidrotermal através de espectrorradiometria e análise de imagens orbitais TM (estudo de caso no Greenstone Belt de Guarinos). Dissertação de Mestrado, Instituto de Geociências, Universidade de Brasília, Brasília, 293p.

Flores J.A.A. 1998. Mineralogia e Geoquímica das alterações metassomáticas e hidrotermais das rochas encaixantes da mineralização de fluorita da mina Rio dos Bugres, Santa Catarina, Brasil. Tese de doutoramento, Instituto de Geociências, Universidade Federal do RioGrande do Sul, $245 \mathrm{p}$.

Flores J.A.A., Lisboa N.A., Baptista P.R.D. 1993. Caracterização morfotectônica e morfoestrutural do sudeste de Santa Catarina. Geociências, Rio Claro, 2(1):61-70.

Fragoso Cesar A.R.S. 1980. O Cráton do Rio de La Plata e o
Cinturão Dom Feliciano no Escudo Uruguaio-Sul-Riograndense. In: SBG, Congresso Brasileiro de Geologia, 31, Balneário Camboriú, Anais, 5:2879-2892.

Hackspaker P. C. \& Flores J.A. 1987. Contribuição sobre o controle estrutural das jazidas de fluorita na estrutura Canela Grande, Sudeste de Santa Catarina. In: Simpósio Sul-Brasileiro de Geologia, 3, Curitiba, SBG, Anais, 2:543-550.

Hoff R. \& Bastos Neto A.C. 2002. Aplicação de classificação supervisionada em imagens orbitais apoiada em espectrorradiometria e integrada com dados estruturais para investigação de feições de alteração hidrotermal no Distrito Fluorítico de Santa Catarina, Brasil. In: SBG, Congresso Brasileiro de Geologia, 41, João Pessoa, Anais, p. 159.

Hoff R. 2002. Integração de dados geológicos, de sensoriamento remoto, espectrorradiométricos e geofísicos aplicada à prospecção de depósitos filoneanos de fuorita hidrotermal no Sudeste de Santa Catarina. Tese de Doutoramento, Instituto de Geociências, UFRGS, Porto Alegre, 235p.

Hoff R., Bastos Neto A C., Rolim S.B.A. 2002. Contribuição do Estudo Aeromagnetométrico e de Imagens Orbitais (TM LANDSAT 5) ao Conhecimento do Arcabouço Geológico do Distrito Fluorítico de Santa Catarina (Brasil) e suas Implicações para a Prospecção de Fluorita. Pesquisas em Geociências, 29(2):37-52.

Hoff R., Rolim S.B.A., Bastos Neto A.C. 2004. Mapeamento aerogamaespectrométrico da alteração hidrotermal associada à mineralização no Distrito Fluorítico de Santa Catarina, Brasil. Revista Brasileira de geofísica (Brasilian Journal of Geophisics), 22(1):45-56.

Horbach R. \& Marimon R. 1982. Contribuição à geologia do distrito de fluorita de Santa Catarina. Bol. Téc. Projeto RADAM-BRASIL, Ser. Geologia. Salvador, 104p.

Jelinek A., Bastos Neto A.C., Lelarge M.L.V., Soliani Jr. E. 1999. Apatite fission track dating of fluorite ore veins from Santa Catarina state, Brazil: a complex hydrothermal evolution. Journal of South American Earth Sciences, 12(4):367-377.

Klein M.A. 1998. Integração de imagens TM e aerogeofísicas para análise lito-estrutural de uma porção da zona de cisalhamento dorsal de canguçu, região da QuitériaVárzea do Capivarita, RS. Dissertação de Mestrado, Centro Estadual de Pesquisas em Sensoriamento Remoto e Meteorologia, UFRGS, Porto Alegre, 120p.

Morgental A. \& Kirchner C.A. 1983. Síntese do mapa previsional do distrito de fluorita de Santa Catarina. In: SBG, Simpósio Sul-Brasileiro de Geologia, 1, Porto Alegre, 
Atas, p. 294-306.

Morgental A. 1984. Projeto Fluorita no Sudeste de Santa Catarina: Etapa 2 - Caracterização dos depósitos de Fluorita. Porto Alegre, DNPM/CPRM, 130p. Disponível em: http://acervo.cprm.gov.br/rpi_cprm/RdrGeral3.htm/. Acessado em 04.12.2008.

Richards J.A. 1986. Remote Sensing Digital Image Analysis. An Introduction. Berlim, Springer-Verlag, 281p.

Rocha F.F.N. 1997. Geologia e geoquímica dos filoes de fluorita da parte norte do Distrito Fluorítico de Santa Catarina. Dissertação de Mestrado, Instituto de Geociências, Universidade Federal do Rio Grande do Sul, Porto Alegre, 188p.

ROCKWARE. 1999. Rockworks 99. RockWare, Inc. Disponível em: http://www.rockware.com/. Acessado em 21.05.2006.

RESEARCH SYSTEMS, INC. (RSI). 2008. Envi 3.5. Eastman Kodak Company. Disponível em: http://www.rsinc. com/. Acessado em 01.07.2007.

Sallet R. 1988. Étude pétrologique et métallogénique d'un secteur du District à Fluorine de Santa Catarina, Brésil. Les granitoïds précambriens monzonitiques source probable de la fluorine filonienne post-jurassique. Thèse de Doctorat, Université de Paris VI, 233p.

Sallet R., Moritz R. \& Fontignie D. 2000. Fluorite ${ }^{87} \mathrm{Sr} /{ }^{86} \mathrm{Sr}$ and REE constraints on fluid-melt relations, crystalization time span and bulk $\mathrm{D}^{\mathrm{Sr}}$ of evolved high-silica granites, Tabuleiro Granite, Santa Catarina, Brazil. Chemical Geology, 164:81-92.

Savi C.N. 1980. Gênese e controle das mineralizações de fluorita da região de Criciúma, SC. Brasília, 112 p. Dissertação de Mestrado, Instituto de Geociências, Universidade de Brasília.

Scheibe L.F. 1986. Geologia e petrologia do Distrito Alcalino de Lages, SC, Brasil. Tese de Doutoramento em Geoquímica, Inst. de Geociências, USP, São Paulo, 224p.

Silva L.C., Hartmann L.A., McNaughton N.J., Fletcher I.R. 1999. SHRIMP U/Pb zircon dating of neoproterozoic granitic magmatism and collision in the Pelotas Batholith, southern Brazil. Geological Survey of Brazil, Belo Horizonte, Brazil. International Geology Review, 41(6):531-551.

Silva M.A.S. \& Leites S.R. (org.) 2000. Programa Levantamentos Geológicos Básicos do Brasil. Criciúma, Folha SH.22-X-B. Estado de Santa Catarina. Escala 1:250000. Brasília, CPRM, CD-Rom.

Teixeira C.A.S. 1969. Relatório de datações geocronológicas do Projeto Básico da Região Leste e do Projeto Específico da Fluorita. Porto Alegre, DNPM. Inédito, 39p.

Zanini L.F.P., Branco P.M., Camozzato E., Ramgrab G.E. 1997. Folha Florianópolis ( $S G$ 22-2-D-V) e Lagoa ( $S G$ 22-2-D-VI)., Santa Catarina (1:100.000). Programa Levantamentos Geológicos Básicos do Brasil. Brasília, CPRM, 259p.

Manuscrito ID 8612

Submetido em 11 de janeiro de 2008 Aceito em 20 de junho de 2008 Sistema eletrônico de submissão 